\title{
STRONGLY DAMPED SEMILINEAR EQUATIONS
}

\author{
D. BAHUGUNA \\ Lucknow University \\ Department of Mathematics \& Astronomy \\ Lucknow 226 007, India
}

(Received October, 1994; Revised February, 1995)

\begin{abstract}
A class of strongly damped semilinear equations is studied by using the theory of analytic semigroups. Conditions (on the nonlinear forcing term) are given under which the existence and uniqueness of local and global classical solutions are ensured.
\end{abstract}

Key words: Strongly Damped Wave Equation, Analytic Semigroup, Classical Solution, Mild Solution.

AMS (MOS) subject classifications: 34G20, 35A07.

\section{Introduction}

Let $\Omega$ be a bounded domain in $\mathbb{R}^{n}$ with sufficiently smooth boundary $\partial \Omega$ and let $L u=\sum_{|\alpha| \leq 2 m}$ $a_{\alpha}(x) D^{\alpha} u$ be a strongly elliptic differential operator of order $2 m$ in $\Omega$. We consider the following strongly damped wave equation

$$
\begin{gathered}
u_{t t}+(a L+b) u_{t}+(c L+d) u=f\left(t, u, u_{t}\right), \text { in } \Omega \times\left(t_{0}, T\right), \\
u\left(x, t_{0}\right)=x_{0} \text { and } u_{t}\left(x, t_{0}\right)=x_{1} \text { for } x \in \Omega, \\
D^{\alpha} u=0 \text { for }(x, t) \in \partial \Omega \times\left[t_{0}, T\right),|\alpha| \leq m-1,
\end{gathered}
$$

where $a>0$. Duvaut and Lions [4], Glowinski, Lions and Tremolieres [5] have studied particular cases of (1.1) in which the $n$-dimensional Laplacian, $L=-\Delta$, in the context of the theorem of viscoelastic materials. For more information on these particular cases, we refer the reader to Webb [8] and Ang and Dinh [1].

We treat (1.1) as a special case of the following abstract second order semilinear differential equation in a Banach space $X$ :

$$
\begin{gathered}
u^{\prime \prime}(t)+(a A+b I) u^{\prime}(t)+(c A+d I) u(t) \\
=f\left(t, u(t), u^{\prime}(t)\right), \quad t>t_{0}, \\
u\left(t_{0}\right)=x_{0} \text { and } u^{\prime}\left(t_{0}\right)=x_{1} .
\end{gathered}
$$

For (1.1) we take $A=L$ and assume that $-A$ generates an analytic semigroup $T(t)$ in $X$ (cf. 
Theorem 7.3.5 in Pazy [6]). The nonlinear map $f$ is supposed to satisfy assumption (F), stated in Section 2.

Sandefur [7] studied the second order semilinear differential equation

$$
\begin{gathered}
u^{\prime \prime}(t)+A u^{\prime}(t)+B u(t)=f\left(t, u(t), u^{\prime}(t)\right) \\
u(0)=\phi, u^{\prime}(0)=\psi
\end{gathered}
$$

in a Banach space $X$ under the assumptions that the linear operators $A$ and $B$ can be decomposed as $-A=A_{1}+A_{2}$ and $B=A_{2} A_{1}$, where $A_{k}$ generates a $c_{0}$-semigroup $T_{k}(t), k=1,2, ;$ and $f$ satisfies a locally Lipschitz condition. He established the local existence and uniqueness of a mild solution to (1.3) which reads as follows. There exists a continuous function $u$ on $[0, c]$ for some $c>0$ such that $u$ satisfies the integral equation

$$
\begin{gathered}
u(t)=T_{1}(t) \phi+\int_{0}^{t} T_{1}(t-\tau) T_{2}(\tau)\left(\psi-A_{1} \phi\right) d \tau \\
+\int_{0}^{t} \int_{0}^{\tau} T_{1}(t-\tau) T_{2}(t-s) f(s, u(s)) d s d \tau
\end{gathered}
$$

where $\phi \in D\left(A_{1}\right)$. Aviles and Sandefur [2] studied the wellposedness of (1.3) under similar conditions.

We notice that, for the existence and uniqueness of solutions to (1.2), it suffices to study the problem

$$
\begin{gathered}
u^{\prime \prime}(t)+A u^{\prime}(t)=f\left(t, u(t), u^{\prime}(t)\right) \\
u\left(t_{0}\right)=x_{0}, \quad u^{\prime}\left(t_{0}\right)=x_{1}
\end{gathered}
$$

where the terms $b u^{\prime}(t)$ and $(c A+d I) u(t)$ are merged with $f$ so that $f$ still satisfies assumption (F).

Engler, Neubrander and Sandefur [3] proved the local existence and uniqueness of a mild solution to (1.4) under the assumptions that $-A$ generates an analytic semigroup $T(t)$ in $X$ and $f$ satisfies a condition similar to assumption (F), where a mild solution on $\left[t_{0}, t_{1}\right)$, for some $t_{1}>t_{0}$, to (1.4) is the first component of a solution $(u(t), v(t))$ of the integral equations

$$
\begin{gathered}
u(t)=x_{0}+\left(T\left(t-t_{0}\right)-I\right)(-A)^{-1} x_{1} \\
+\int_{t_{0}}^{t}(T(t-s)-I)(-A)^{-1} f(s, u(s), v(s)) d s, \quad t_{0} \leq t<t_{1} \\
v(t)=T\left(t-t_{0}\right) x_{1}+\int_{t_{0}}^{t} T(t-s) f(s, u(s), v(s)) d s, t_{0} \leq t<t_{1} .
\end{gathered}
$$

We improve the result of [3] by showing that (1.4) has a unique classical solution locally, i.e., there exists a unique $u \in C^{1}\left(\left[t_{0}, t_{1}\right): X\right) \cap C^{2}\left(\left(t_{0}, t_{1}\right): X\right)$ and satisfies (1.4) on $\left[t_{0}, t_{1}\right)$ for some $t_{1}>t_{0}$. Further, we discuss the continuation of this solution, maximal interval of existence and the global existence. We achieve these objectives by extending the ideas and techniques used in the proofs of Theorems 6.3.1 and 6.3.3 in Pazy [6] concerning the semilinear equations of the first order to problem (1.4). For the global existence, we require a modified version of Lemma 4.1, stated and proved at the end of Section 4 originally stated in Pazy [6] as Lemma 5.6.7. 


\section{Preliminaries and Assumptions}

Let $X$ be a Banach space and let $-A$ generate the analytic semigroup $T(t)$ in $X$. We note that if $-A$ is the infinitesimal generator of an analytic semigroup then $-(A+\alpha I)$ is invertible and generates a bounded analytic semigroup for $\alpha>0$ large enough. This allows us to reduce the general case, in which $-A$ is the infinitesimal generator of an analytic semigroup, to the case where the semigroup is bounded and the generator is invertible. Hence, for convenience, without loss of generality, we assume that $T(t)$ is bounded, that is $\|T(t)\| \leq M$ for $t \geq 0$ and $0 \in \rho(-A)$, i.e., $-A$ is invertible. Here $\rho(-A)$ is the resolvent set of $-A$. It follows that, for $0 \leq \alpha \leq 1, A^{\alpha}$ can be defined as a closed linear invertible operator with its domain $D\left(A^{\alpha}\right)$ being dense in $X$. We denote by $X_{\alpha}$ the Banach space $D\left(A^{\alpha}\right)$ equipped with the norm $\|x\|_{\alpha}=$ $\left\|A^{\alpha} x\right\|$ which is equivalent to the graph norm of $A^{\alpha}$. For $0<\alpha<\beta$, we have $X_{\beta} \subset X_{\alpha}$ and the embedding is continuous.

We consider the problem

$$
\begin{gathered}
u^{\prime \prime}(t)+A u^{\prime}(t)=f\left(t, u(t), u^{\prime}(t)\right) \\
u\left(t_{0}\right)=x_{0}, \quad u^{\prime}\left(t_{0}\right)=x_{1}
\end{gathered}
$$

Regarding the function $f$ we make the following

Assumption (F): Let $U$ be an open set of $\mathbb{R}_{+} \times X_{1} \times X_{\alpha}$. A function $f$ is said to satisfy the assumption (F) if for every $(t, x, \tilde{x}) \in U$ there exist a neighborhood $V \subset U$ and constants $L \geq 0$, $0<\vartheta \leq 1$, such that

$$
\left\|f\left(t_{1}, x_{1}, \tilde{x}_{1}\right)-f\left(t_{2}, x_{2}, \tilde{x}_{2}\right)\right\| \leq L\left[\left|t_{1}-t_{2}\right|^{\vartheta}+\left\|x_{1}-x_{2}\right\|_{1}+\left\|\widetilde{x}_{1}-\widetilde{x}_{2}\right\|_{\alpha}\right]
$$

for all $\left(t_{i}, x_{i}, \tilde{x}_{i}\right) \in V$.

By a local classical solution to (2.1) we mean a function $u \in C^{1}\left(\left[t_{0}, t_{1}\right): X\right) \cap C^{2}\left(\left(t_{0}, t_{1}\right): X\right)$ satisfying $(2.1)$ on $\left[t_{0}, t_{1}\right)$ for some $t_{1}>t_{0}$. By a local mild solution to (2.1) we mean the first component of a solution $(u, v)$ to the integral equations $(1.5)$ on $\left[t_{0}, t_{1}\right)$ for some $t_{1}>t_{0}$.

\section{Local Existence}

As we have already pointed out, without loss of generality, the semigroup, generated by $-A$, can be assumed to be bounded and $A$ invertible. Under these conditions imposed on $A$ we prove the following local existence and uniqueness theorem.

Theorem 3.1: Suppose that $-A$ generates the analytic semigroup $T(t)$ such that $\|T(t)\| \leq M$ and $0 \in \rho(-A)$. If the map $f$ satisfies assumption $(\mathrm{F})$ then (2.1) has a unique local classical solution.

Proof: Fix $\left(t_{0}, x_{0}, x_{1}\right)$ in $U$ and choose $t_{1}^{\prime}>t_{0}$ and $\delta>0$ such that (2.2), with some fixed constants $L \geq 0$ and $0<\vartheta \leq 1$, holds on the set

$$
V=\left\{(t, x, \tilde{x}) \in U: T_{0} \leq t \leq t_{1}^{\prime},\left\|x-x_{0}\right\|_{1}+\left\|\widetilde{x}-x_{1}\right\|_{\alpha} \leq \delta\right\} .
$$

Let $B=\max _{t_{0} \leq t \leq t_{1}^{\prime}}\left\|f\left(t, x_{0}, x_{1}\right)\right\|$. Choose $t_{1}>t_{0}$ such that

$$
\begin{aligned}
& t_{0} \leq t \leq t_{1}^{\prime} \\
& t_{1}-t_{0}<\min \left\{t_{1}^{\prime}-t_{0}, \frac{\delta}{3}(1+M)^{-1}(L \delta+B)^{-1},\left[\frac{\delta}{3} C_{\alpha}^{-1}(1-\alpha)(L \delta+B)^{-1}\right]^{\frac{1}{1-\alpha}}\right\}
\end{aligned}
$$


where $C_{\alpha}$ is a positive constant depending on $\alpha$ and satisfying

$$
\left\|A^{\alpha} T(t)\right\| \leq C_{\alpha} t^{-\alpha} \text { for } t>0 \text {. }
$$

Let $Y=C\left(\left[t_{0}, t_{1}\right]: X \times X\right)$. Then $y \in Y$ is of the form $y=\left(y_{1}, y_{2}\right), y_{i} \in C\left(\left[t_{0}, t_{1}\right]: X\right), i=1,2 . Y$, endowed with the supremum norm,

$$
\left\|\left(y_{1}, y_{2}\right)\right\|_{Y}=\sup _{t_{0} \leq t \leq t_{1}}\left[\left\|y_{1}(t)\right\|+\left\|y_{2}(t)\right\|\right]
$$

is a Banach space. We define a map $F$ on $Y$ by $F y=F\left(y_{1}, y_{2}\right)=\left(\widehat{y}_{1}, \widehat{y}_{2}\right)$ with

$$
\begin{gathered}
\widehat{y}_{1}(t)=A x_{0}-\left(T\left(t-t_{0}\right)-I\right) x_{1}-\int_{t_{0}}^{t}(T(t-s)-I) f_{y}(s) d s \\
\widehat{y}_{2}(t)=T\left(t-t_{0}\right) A^{\alpha} x_{1}+\int_{t_{0}}^{t} T(t-s) A^{\alpha} f_{y}(s) d s
\end{gathered}
$$

where $f_{y}(t)=f\left(t, A^{-1} y_{1}(t), A^{-\alpha} y_{2}(t)\right)$ for $t \in\left[t_{0}, t_{1}\right]$.

For every $y \in Y, F y\left(t_{0}\right)=\left(A x_{0}, A^{\alpha} x_{1}\right)$, and the assumption (F) on $f$ implies that $F: Y \rightarrow Y$. Let $S$ be a nonempty closed and bounded set given by

$$
\begin{gathered}
S=\left\{y \in Y: y=\left(y_{1}, y_{2}\right), y_{1}\left(t_{0}\right)=A x_{0}, y_{2}\left(t_{0}\right)=A^{\alpha} x_{1},\right. \\
\left.\left\|y_{1}(t)-A x_{0}\right\|+\left\|y_{2}(t)-A^{\alpha} x_{1}\right\| \leq \delta\right\} .
\end{gathered}
$$

Let $y=\left(y_{1}, y_{2}\right)$ be any element of $S$. Inside the integrals in (3.4) we add and subtract $f\left(s, x_{0}, x_{1}\right)$ and use the fact that $\|T(t)\| \leq M$ and (3.3) to obtain

$$
\begin{gathered}
\left\|y_{1}(t)-A x_{0}\right\|+\left\|y_{2}(t)-A^{\alpha} x_{1}\right\| \\
\leq \frac{\delta}{3}+(1+M)(L \delta+B)\left(t_{1}-t_{0}\right)+C_{\alpha}(1-\alpha)^{-1}(L \delta+B)\left(t_{1}-t_{0}\right)^{\frac{1}{1-\alpha}} \leq \delta .
\end{gathered}
$$

Hence $F: S \rightarrow S$. Now let $\left(y_{1}, y_{2}\right)$ and $\left(z_{1}, z_{2}\right)$ be any two elements of $S$. We use assumption (F) to get

$$
\begin{gathered}
\left\|\widehat{y}_{1}(t)-\widehat{z}_{1}(t)\right\|+\left\|\widehat{y}_{2}(t)-\widehat{z}_{2}(t)\right\| \\
\leq\left[L(1+M)\left(t_{1}-t_{0}\right)+L C_{\alpha}(1-\alpha)^{-1}\left(t_{1}-t_{0}\right)^{\frac{1}{1-\alpha}}\right]\left\|\left(y_{1}, y_{2}\right)-\left(z_{1}, z_{2}\right)\right\|_{Y} \\
\leq \frac{2}{3}\left\|\left(y_{1}, y_{2}\right)-\left(z_{1}, z_{2}\right)\right\|_{Y} .
\end{gathered}
$$

Thus $F$ is a contraction on $S$. Therefore, it has a unique fixed point in $S$. Let $\bar{y}=\left(\bar{y}_{1}, \bar{y}_{2}\right) \in S$ be that fixed point of $F$. Then,

$$
\bar{y}_{1}(t)=A x_{0}-\left(T\left(t-t_{0}\right)-I\right) x_{1}-\int_{t_{0}}^{t}(T(t-s)-I) f_{\bar{y}}(s) d s
$$




$$
\bar{y}_{2}(t)=T\left(t-t_{0}\right) A^{\alpha} x_{1}+\int_{t_{0}}^{t} T(t-s) A^{\alpha} f_{\bar{y}}(s) d s,
$$

where $f_{\bar{y}}(t)=f\left(t, A^{-1} \bar{y}_{1}(t), A^{-\alpha} \bar{y}_{2}(t)\right)$. We note that $(u, v)=\left(A^{-1} \bar{y}_{1}, A^{-\alpha} \bar{y}_{2}\right)$ is the unique solution of integral equations (1.5) on $\left[t_{0}, t_{1}\right]$. Assumption (F) and the continuity of $\bar{y}_{1}$ and $\bar{y}_{2}$ on $\left[t_{0}, t_{1}\right]$ imply that the map $t \mapsto f_{\bar{y}}(t)$ is continuous and hence bounded on $\left[t_{0}, t_{1}\right]$. Let $\left\|f_{\bar{y}}(t)\right\| \leq N$ for $t_{0} \leq t \leq t_{1}$. We will now show that $t \mapsto f_{\bar{y}}(t)$ is locally Hölder continuous on $\left(t_{0}, t_{1}\right]$. For this we first show that $\bar{y}_{1}$ and $\bar{y}_{2}$ are locally Hölder continuous on $\left(t_{0}, t_{1}\right]$. From Theorem 2.6.13 in Pazy [6], for every $0<\beta<1-\alpha$ and every $0<h<1$, we have

$$
\left\|(T(h)-I) A^{\alpha}(t-s)\right\| \leq C_{\beta} h^{\beta}\left\|A^{\alpha+\beta} T(t-s)\right\| \leq C_{\beta} H^{\beta}(t-s)^{-(\alpha+\beta)} .
$$

Now,

We use (3.6) to get

$$
\begin{aligned}
\left\|\bar{y}_{2}(t+h)-\bar{y}_{2}(t)\right\| & \leq\left\|(T(h)-I) A^{\alpha} T\left(t-t_{0}\right) x_{1}\right\|+\int_{t_{0}}^{t}\left\|(T(h)-I) A^{\alpha} T(t-s) f_{\bar{y}}(s)\right\| d s \\
& +\int_{t}^{t+h}\left\|A^{\alpha} T(t+h-s) f_{\bar{y}}(s)\right\| d s=I_{1}+I_{2}+I_{3} .
\end{aligned}
$$

$$
\begin{gathered}
I_{1} \leq C\left(t-t_{0}\right)^{-(\alpha+\beta)} h^{\beta} \leq M_{1} h^{\beta}, \\
I_{2} \leq N h^{\beta} C_{\beta} \int_{t_{0}}^{t}(t-s)^{-(\alpha+\beta)} d s \leq M_{2} h^{\beta}, \\
I_{3} \leq N C_{\alpha} \int_{t}^{t+h}(t+h-s)^{-\alpha} d s \leq M_{3} h^{\beta} .
\end{gathered}
$$

Here $M_{1}$ depends on $t$ and increases to infinity as $t \downarrow t_{0}$, while $M_{2}$ and $M_{3}$ can be chosen independent of $t$. From the above estimates, it follows that there exists a positive constant $C$ such that for every $t_{0}^{\prime}>t_{0}$

$$
\left\|\bar{y}_{2}(t)-\bar{y}_{2}(s)\right\| \leq C|t-s|^{\beta} \text { for } t_{0}<t_{0}^{\prime} \leq t, s \leq t_{1} .
$$

Similar result holds for $\bar{y}_{1}$ (if we take $\alpha=0$ in the above considerations). The local Hölder continuity of $f_{\bar{y}}(t)$ on $\left(t_{0}, t_{1}\right]$ follows from assumption $(\mathrm{F})$ and the local Hölder continuity of $\bar{y}_{1}$ and $\bar{y}_{2}$ on $\left(t_{0}, t_{1}\right]$.

Consider the initial value problem

$$
\begin{gathered}
\frac{d v}{d t}(t)+A v(t)=f_{\bar{y}}(t) \\
v\left(t_{0}\right)=x_{1} .
\end{gathered}
$$

By Corollary 4.3.3 in Pazy [6], (3.7) has a unique solution $v \in C^{1}\left(\left(t_{0}, t_{1}[: X)\right.\right.$ given by 


$$
v(t)=T\left(t-t_{0}\right) x_{1}+\int_{t_{0}}^{t} T(t-s) f_{\bar{y}}(s) d s .
$$

For $t>t_{0}$, each term on the right-hand side belongs to $D(A)$ and hence belongs to $D\left(A^{\alpha}\right)$. Application of $A^{\alpha}$ to both the sides in (3.8) and the uniqueness of $\bar{y}_{2}$ imply that $A^{\alpha} v(t)=\bar{y}_{2}(t)$ or $v(t)=A^{-\alpha} \bar{y}_{2}(t)$. Let $u(t)=A^{-1} \bar{y}_{1}(t)$. Then, we have $u(t)=x_{0}+\int_{t_{0}}^{t} v(s) d s$ which yields $u(t) \in$ $C^{1}\left(\left[t_{0}, t_{1}\right): X\right) \cap C^{2}\left(\left(t_{0}, t_{1}\right): X\right)$. Thus, $u$ satisfies $(2.1)$ on $\left[t_{0}, t_{1}\right)$. This completes the proof of Theorem 3.1.

Remark 3.1: Theorem 3.1 can be applied to assert that a unique local mold solution of the general linear second order equation established by Engler, Neubrander and Sandefur [3] in Example 3.1, is in fact a local classical solution. Using arguments of [3], this solution can be proved to be global.

Remark 3.2: Theorem 3.1 can also be used to prove the regularity of local mild solutions to the strongly damped quasilinear wave equations and the strongly damped Klein-Gordon equation of Examples 3.2 and 3.3 in [3], respectively.

\section{Global Existence}

In [3] it was proven that if $f:[0, \infty) \times X_{1} \times X_{\alpha} \rightarrow X$ satisfies assumption (F) globally, then the solution $(u, v)$ to integral equations (1.5) can be continued to the maximal interval of existence $[0, T)$, and, if $T<\infty$, then $\varlimsup_{t \rightarrow T-}\left[\|u(t)\|_{1}=\|v(t)\|_{\alpha}\right]=+\infty$.

We prove the following global existence result.

Theorem 4.1: Let $0 \in D(-A)$ and let $-A$ be the infinitesimal generator of an analytic semigroup $T(t)$ such that $\|T(t)\| \leq M$ for $t \geq 0$. Let $f:[0, \infty) \times X_{1} \times X_{\alpha} \rightarrow X$ satisfy assumption (F). If there exists a nondecreasing function $k:\left[t_{0}, \infty\right) \rightarrow \mathbb{R}_{+}$, such that

$$
\|f(t, x, \tilde{x})\| \leq k(t)\left[1+\|x\|_{1}+\|\tilde{x}\|_{\alpha}\right] \text { for } t \geq t_{0},(x, \tilde{x}) \in X_{1} \times X_{\alpha}
$$

then for each $\left(x_{0}, x_{1}\right) \in X_{1} \times X_{\alpha},(2.1)$ has a unique classical solution $u$ which exists for all $t \geq t_{0}$.

Proof: Let $\left[t_{0}, T\right)$ be the maximal interval of existence for the solution $u$ to (2.1) guaranteed by Theorem 3.1. It suffices to prove that $\left[\|u(t)\|_{1}+\|v(t)\|_{\alpha}\right] \leq C$ on $\left[t_{0}, T\right)$ for some fixed constant $C \geq 0$ independent of $t$.

Now, since $u(t)$ is a solution of $(2.1)$ on $\left[t_{0}, T\right)$, it is also a mild solution to (2.1). Therefore, from (3.5), we have

$$
\begin{gathered}
A u(t)=A x_{0}-\left(T\left(t-t_{0}\right)-I\right) x_{1}-\int_{t_{0}}^{t}(T(t-s)-I) \bar{f}(s) d s \\
A^{\alpha} u^{\prime}(t)=T\left(t-t_{0}\right) A^{\alpha} x_{1}+\int_{t_{0}}^{t} T(t-s) A^{\alpha} \bar{f}(s) d s
\end{gathered}
$$

where $\bar{f}(t)=f\left(t, u(t), u^{\prime}(t)\right)$ for $t \in\left[t_{0}, T\right)$. Our assumptions on $f$ imply that

$$
\left[1+\|u(t)\|_{1}+\left\|u^{\prime}(t)\right\|_{\alpha}\right]
$$




$$
\begin{gathered}
\leq C_{1}+C_{2} \int_{t_{0}}^{t}\left[1+\|u(s)\|_{1}+\left\|u^{\prime}(s)\right\|_{\alpha}\right] d s \\
+C_{3} \int_{t_{0}}^{t}(t-s)^{-\alpha}\left[1+\|u(s)\|_{1}+\left\|u^{\prime}(s)\right\|_{\alpha}\right] d s .
\end{gathered}
$$

Now, we only need to apply the following lemma to get the required estimate.

Lemma 4.1: Let $\phi(t, s) \geq 0$ be continuous on $0 \leq s<t \leq T$. If there are positive constants $A, B_{1}, B_{2}$ and $\beta$, such that

$$
\phi(t, s) \leq A+B_{1} \int_{s}^{t} \phi(\sigma, s) d \sigma+B_{2} \int_{s}^{t}(t-\sigma)^{\beta-1} \phi(\sigma, s) d \sigma,
$$

for $0 \leq s<t \leq T$, then there exists a positive constant $C$ such that $\phi(t, s) \leq C$ for $0 \leq s<t \leq T$.

Proof of Lemma 4.1: We have

$$
\int_{s}^{t} \int_{s}^{\sigma}(t-\sigma)^{\beta-1} \phi(\tau, s) d \tau d \sigma=\int_{s}^{t}\left(\int_{\tau}^{t}(t-\sigma)^{\beta-1} d \sigma\right) \phi(\tau, s) d \tau
$$

and the well-known identity

$$
\int_{\tau}^{t}(t-\sigma)^{\beta-1}(\sigma-\tau)^{\gamma-1} d \sigma=(t-\tau)^{\beta+\gamma-1} \frac{\Gamma(\beta) \Gamma(\gamma)}{\Gamma(\beta+\gamma)} .
$$

Iterating (3.9) $n-1$ times using (3.10) and (3.11) and majorating $(t-s)$ and $(t-\tau)$ by $T$, we get

$$
\begin{aligned}
\phi(t, s) \leq & A \sum_{j=0}^{n-1}\left(\frac{B_{2} T^{\beta}}{\beta}\right)^{j}+B_{1} \sum_{j=0}^{n-1}\left(\frac{B_{2} T^{\beta}}{\beta}\right)^{j} \int_{s}^{t} \phi(\sigma, s) d \sigma \\
& +\frac{\left(B_{2} \Gamma(\beta)\right)^{n}}{\Gamma(n \beta)} \int_{s}^{t}(t-\sigma)^{n \beta-1} \phi(\sigma, s) d \sigma .
\end{aligned}
$$

Choosing $n$ sufficiently large so that $n \beta>1$ and replacing $(t-\sigma)^{n \beta-1}$ by $T^{n \beta-1}$ we get

$$
\phi(t, s) \leq c_{1}+c_{2} \int_{s}^{t} \phi(\sigma, s) d \sigma
$$

where $c_{1}$ and $c_{2}$ are positive constants independent of $s$. The required result then follows from the Gronwall's inequality. This ends the proof of the lemma and of Theorem 4.1.

Remark 4.1: Theorem 4.1 gives the global existence and uniqueness of a classical solution to the Klein-Gordon equation considered in Example 3.3 in [3] (cf. also Example 4.1 in [3]).

\section{Acknowledgement}

The author wishes to thank the referee for his/her helpful suggestions. 


\section{References}

[1] Ang, D.D. and Dinh, A.P.N., On strongly damped wave equation $u_{t t}-\Delta u_{t}-\Delta u+f(u)=$ 0, SIAM J. Math. Anal. 19 (1988), 1409-1418.

[2] Aviles, P. and Sandefur, J., Nonlinear second order equations with applications to partial differential equations, J. Diff. Eqns. 58 (1985), 404-427.

[3] Engler, H., Neubrander, F. and Sandefur, J., Strongly damped semilinear second order equations, In: Proc. of the First Howard Univ. Symp. on Nonlinear Semigroups, Partial Diff. Eqns. and Attractors, Springer-Verlag Lecture Notes in Math 1248 (1985), 52-62.

[4] Duvaut, G. and Lions, J.L., Les Inequations en Mecanique Physique, Dunod, Paris 1972.

[5] Glowinski, R., Lions, J.L. and Tremolieres, R., Analyse Numerique des Inequations Variationnelles, Dunod, Paris 1976.

[6] Pazy, A., Semigroups of Linear Operators and Applications to Partial Differential Equations, Springer-Verlag, New York 1983.

[7] Sandefur, J.T., Existence and uniqueness of solutions of second order nonlinear differential equations, SIAM J. Math. Anal. 14 (1983), 477-487.

[8] Webb, G.F., Existence and asymptotic behavior for a strongly damped nonlinear wave equation, Can. J. Math. 32 (1980), 631-643. 


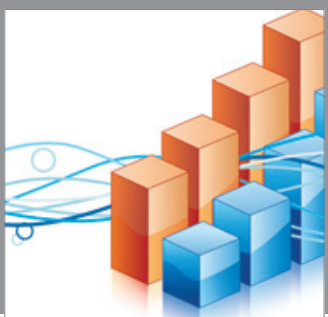

Advances in

Operations Research

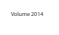

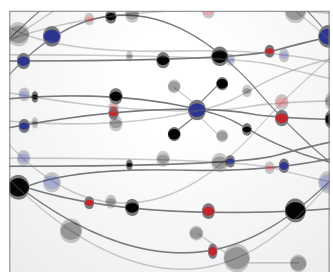

\section{The Scientific} World Journal
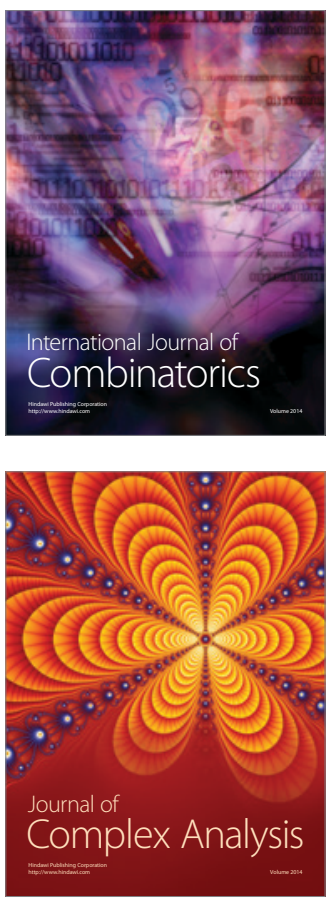

International Journal of

Mathematics and

Mathematical

Sciences
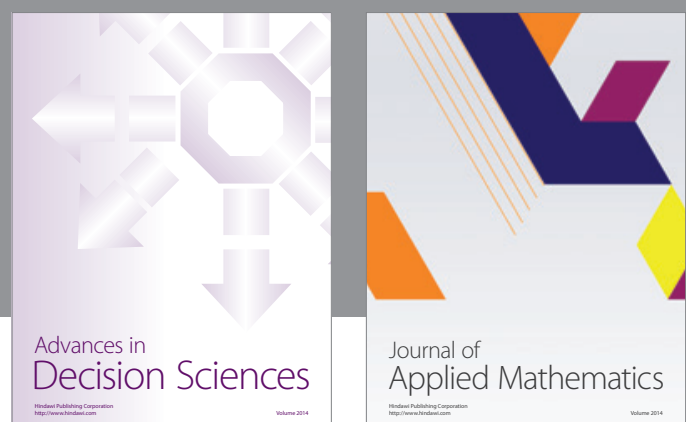

Journal of

Applied Mathematics
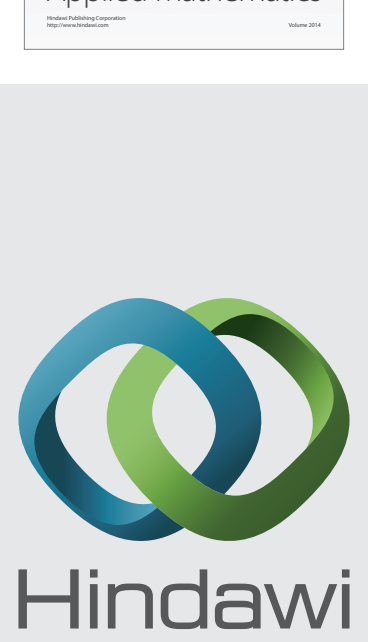

Submit your manuscripts at http://www.hindawi.com
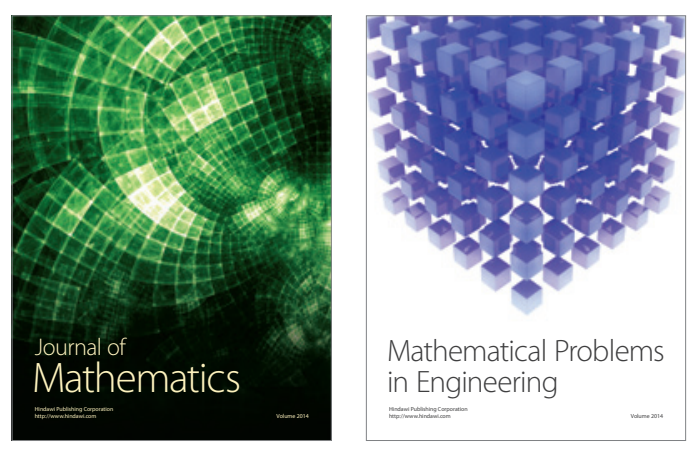

Mathematical Problems in Engineering
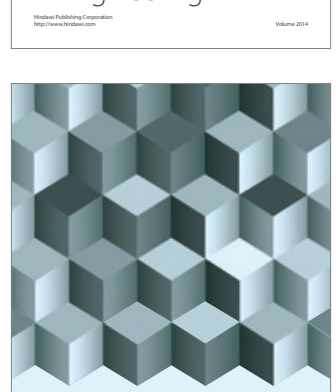

Journal of

Function Spaces
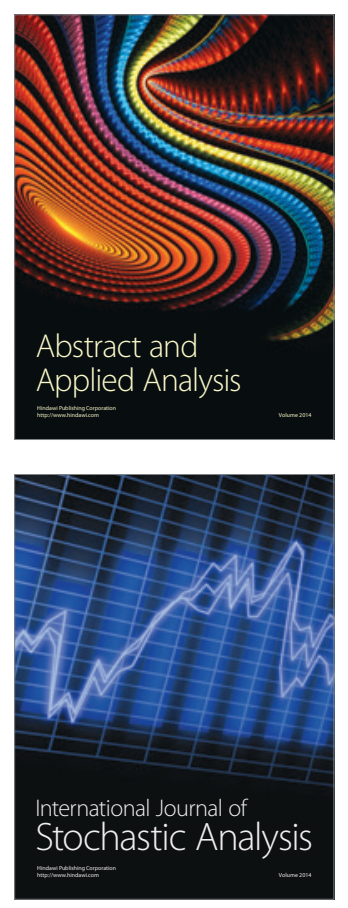

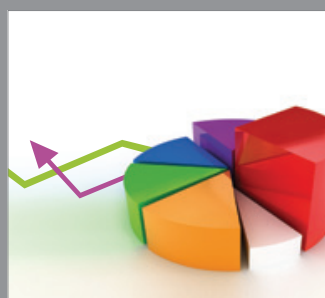

ournal of

Probability and Statistics

Promensencen
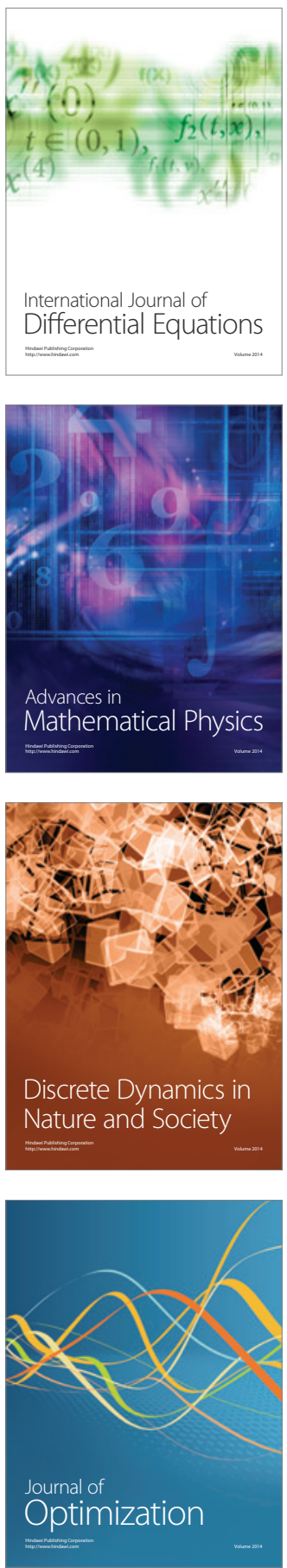